\title{
An Improved Gravitational Coefficient Function for Enhancing Gravitational Search Algorithm's Performance
}

\author{
Pattrawet Tharawetcharak, Thanathorn Karot, and Choosak Pornsing
}

\begin{abstract}
This article proposes a new gravitational coefficient function of the gravitational search algorithm (GSA). Since the function concerns to the performance of GSA, we investigate its characteristic which influences the algorithm on global search performance. The novel function is compared to a former function in literature on four benchmark functions which incorporated of both unimodal landscape functions and multimodal landscape functions. The experimental results show that the proposed gravitational coefficient function outperforms the conventional one. The proposed function also shows that it works well on multimodal landscape functions. By balancing between exploration phase and exploitation phase, the slow convergence rate is compensated by the better solutions.
\end{abstract}

Index Terms-GSA, metaheuristics, optimization problems, search performance.

\section{INTRODUCTION}

As the Newtonian law of motion states: "Every particle in the universe attracts every other particle with a force that is directly proportional to the product of their masses and inversely proportional to the square of the distance between them" [1], Rashedi et al. [2] proposed a novel metaheuristics based on the Newton's law of universal gravitation, named Gravitational Search Algorithm (GSA). Since the debut of the seminal article, there are a number of reports about exploiting GSA on many circumstances: such as data analysis [3], energy and renewable energy management [4]-[6], irrigation management [7], robot path planning [8], and logistics management [9].

Besides, the algorithm itself also has been improved in order to cope with particular problems and better performances. Yazdani et al. [10] improved the conventional GSA by dividing the whole swarm (masses) into many sub-swarms and also proposed three strategies for maintaining the communication between sub-swarms. The authors showed that the new approach outperformed competitive algorithms on multimodal functions. Eldos and

Manuscript accepted November 10, 2018; revised April 28, 2019. This work was support in part by Department of Industrial Engineering and Management, Faculty of Engineering and Industrial Technology, Silpakorn University, Nakhon Pathom, Thailand.

Pattrawet Tharawetcharak is with Office of Learning Promotion and Provision Academic Services, Varaya Alongkorn Rajabhat University under The Royal Patronage, Pathumthani, 13180 Thailand and he is also with the Department of Industrial Engineering and Management, Faculty of Engineering and Industrial Technology, Silpakorn University, Nakhon Pathom, 73000 Thailand (e-mail: pattrawet@vru.ac.th).

Thanathorn Karot and Choosak Pornsing are with the Department of Industrial Engineering and Management, Faculty of Engineering and Industrial Technology, Silpakorn University, Nakhon Pathom, 73000 Thailand (e-mail: karot_t@su.ac.th, pornsing_c@su.ac.th).
Al Qasim [11] adapted the transition functions for seeking a better balance between exploration and exploitation. The proposed method was compared with a genetic algorithm (GA). The results showed that its performance dominated the performance of GA. Nevertheless, the author did not discuss the benefits of their improved methods against the conventional GSA.

Yin et al. [12] improved the gravitational search algorithm (GSA) with crossover operation, it is briefly called CROGSA. The algorithm executes the crossover based search scheme to update the position of each solution. The proposed method cultivated the performance of crossover technique to explore the global optimal solution which encouraged the algorithm's performance. Experimentally, the algorithm was better than other optimization approaches. Furthermore, this algorithm is suitable for complex optimization problems.

Khatibinia, \& Khosravi [13] improved gravitational search algorithm and orthogonal crossover (IGSA-OC). The algorithm was verified the efficiency, by benchmarking functions in literatures. The solutions of the IGSA-OC were compared to those of the conventional gravitational search algorithm (GSA), another improved gravitational search algorithm (IGSA), and particle swarm optimization (PSO). The results substantiated that the proposed IGSA-OC significantly performed better than the conventional GSA, IGSA and PSO.

Xiao et al. [14] combined the gravitational search algorithm (GSA) and particle swarm optimization (PSO) to solve a problem of green-based partner selection. The improved method falls into hybridization techniques by adopting a co-evolutionary technique of PSO to GSA, named I-GSA/PSO. The computational experiments showed that I-GSA/PSO yielded better solutions than other competitive ones; especially, on both conventional GSA and PSO.

Even there are a number of fancy GSA variants, we are interested by the impact and performance of gravitational coefficient function of GSA. In this study, we propose a novel gravitational coefficient function in order to enhance the GSA's performance on global search. The reset of this article is organized as follows. Section II described the conventional gravitational search algorithm and its procedure. The conventional gravitational coefficient function is also presented in this section. Our proposed gravitational coefficient function is described in Section III, where we also explain the idea of us on this new function. Section IV illustrates the performance of the proposed function by a computational experiment. The results are compared and discussed in Section V. Finally, the conclusion is drawn in Section VI. 


\section{THE CONVENTIONAL GSA}

The gravitational search algorithm is a kind of population based stochastic search algorithm which was first proposed by Rashedi et al. [1], [2]. The method is based on Newton's theory. As mentioned, Newton's law states that every particle (mass) attracts another particle by means of some gravitational force. Technically, in GSA, each particle has associated with four attributes: particle position, its inertial mass, active gravitational mass, and passive gravitational mass. The particle's position gives us the solution of a problem while fitness function is used to calculate the gravitational and inertial masses [15].

Step by step procedure of Gravitation Search Algorithm is given below and Fig. 1 shows the process flow of GSA:

Step 1: Identification of search space.

Step 2: Generate initial population.

Step 3: Evaluate fitness function for each particle in population.

Step 4: Update the gravitational coefficient value.

$$
\begin{aligned}
G(t) & =G\left(G_{0}, t\right), \\
\operatorname{Best}(t) & =\min _{i \in\{1, \ldots, N\}} F_{i t_{i}}(t) \\
\operatorname{Worst}(t) & =\max _{i \in\{1, \ldots, N\}} F_{i t_{i}}(t)
\end{aligned}
$$

Step 5: Calculate the total force in different direction $\left(M_{i}\right)$ and acceleration $\left(a_{i}\right)$ by using:

$$
\begin{gathered}
M_{i}(t)=\frac{m_{i}(t)}{\sum_{j=1}^{N} m_{i}(t)}, \text { where } m_{i}=\frac{F i t_{i}(t) \text {-Worst }(t)}{\text { Best }(t) \text {-Worst }(t)} \\
a_{i}^{d}(t)=\frac{F_{i}^{d}(t)}{M_{i j}(t)},
\end{gathered}
$$

where $F_{i}^{d}(t)=\sum_{j=1, i \neq j}^{N} \operatorname{rand}_{j} F_{i j}^{d}(t)$ and $F_{i j}^{d}(t)=$ $G(t) \frac{M_{a j}(t)}{R_{i j}(t)+\varepsilon}\left(x_{j}^{d}(t)-x_{i}^{d}(t)\right)$

where $M_{a j}$ is the active gravitational mass related to agent $j$, $\varepsilon$ is a small constant, $R_{i j}$ is the Euclidian distance between agent $i$ and $j$ and rand $_{j}$ is a uniform random variable in the interval $[0,1]$.

Step 6: Update the particle velocity and position. Velocity and position of a particle is calculated by the following equations:

$$
\begin{gathered}
v_{i}^{d}(t+1)=\operatorname{rand}_{i} \times v_{i}^{d}(t)+a_{i}^{d}(t), \\
x_{i}^{d}(t+1)=x_{i}^{d}(t)+v_{i}^{d}(t+1),
\end{gathered}
$$

where $\operatorname{rand}_{i}$ is a uniform random variable in the interval $[0,1]$.

Step 7: Repeat step 3 through step 6 until the stopping criteria is met.

In our study, we are interested by the gravitational coefficient function, $G(t)$, in step 4 which is the time varying function. We will introduce a new concept of the time varying gravitational coefficient function and illustrate its performance by computational experiments.
From the seminal article of GSA [1], the gravitational coefficient function, $G(t)$, is a decreasing function as shown in (1).

$$
G(t)=G\left(t_{0}\right) \times\left(\frac{t_{0}}{t}\right)^{\beta}, \beta<1
$$

where $G\left(t_{0}\right)$ is the value of the gravitational constant at the first cosmic quantum-interval of time $t_{0} . G(t)$ is the value of the gravitational constant at time $t$ [16]. Fig. 2 shows the values of $G(t)$ when $t$ is varied.

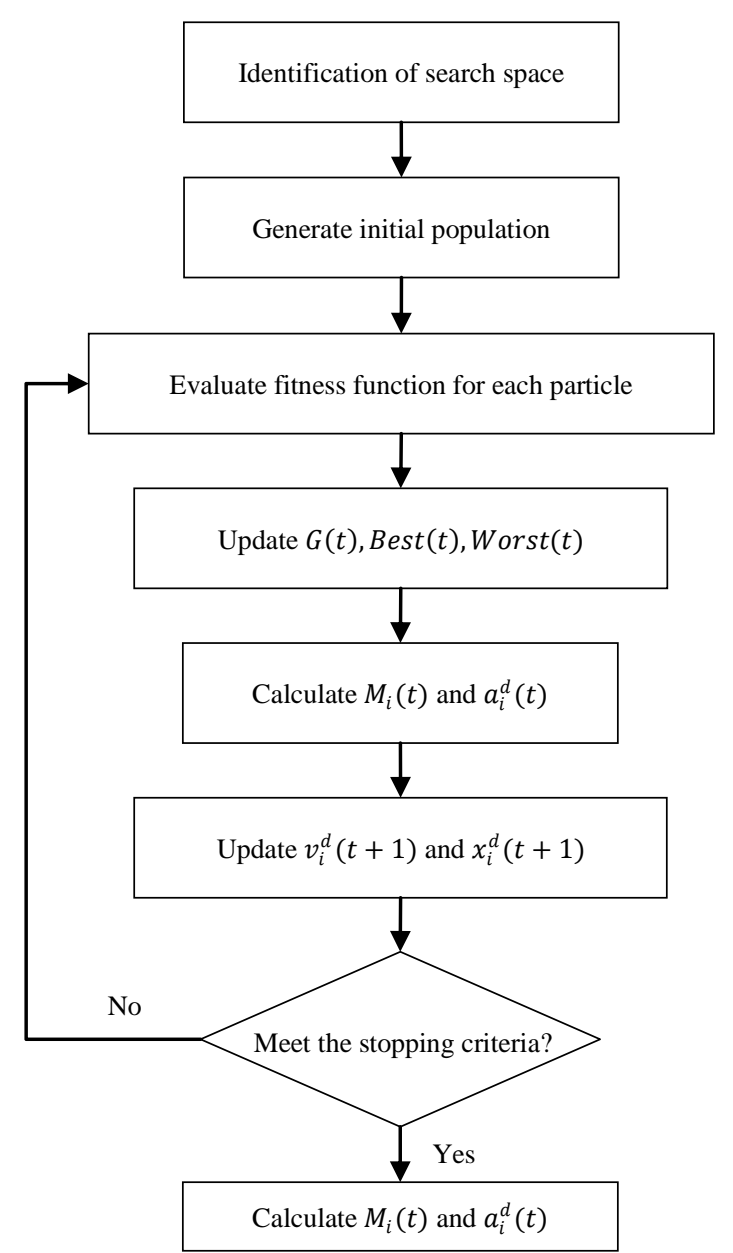

Fig. 1. The procedure of conventional GSA.

\section{The Proposed $G(t)$ FunCtion}

As mentioned earlier, we are interested by the gravitational coefficient function in which we will approach it in different perspective of thinking. As shown in Fig. 2, the function gives the value of $G(t)$ in decreasing line. However, if the search procedure is divided into two phases, we suspect that the $G(t)$ should be small at the first phase and be large in the second phase. The reason behind this idea is that the agents should perform the exploration over the search space, and they should perform the exploitation over the best solution so far.

As a result, we deploy an exponential function based on the conventional gravitational coefficient function, as shown in (2).

$$
G(t)=G\left(t_{0}\right) \exp ^{(t / T)^{\beta}}
$$

where $t$ is the present iteration number, $T$ is the maximum iteration number, and $\beta$ is the constant number. 
Refer to (2), the equation is an increasing function of $t$ and the characteristic of the function depends on the value of $\beta$. Fig. 3 shows the characteristic of the function based on different value of $\beta$. According to Fig. 3, the function closes to linear function when $\beta$ approaches to zero. Besides, the function closes to nonlinear increasing function when $\beta$ gets larger number; and, this characteristic is in our intention.

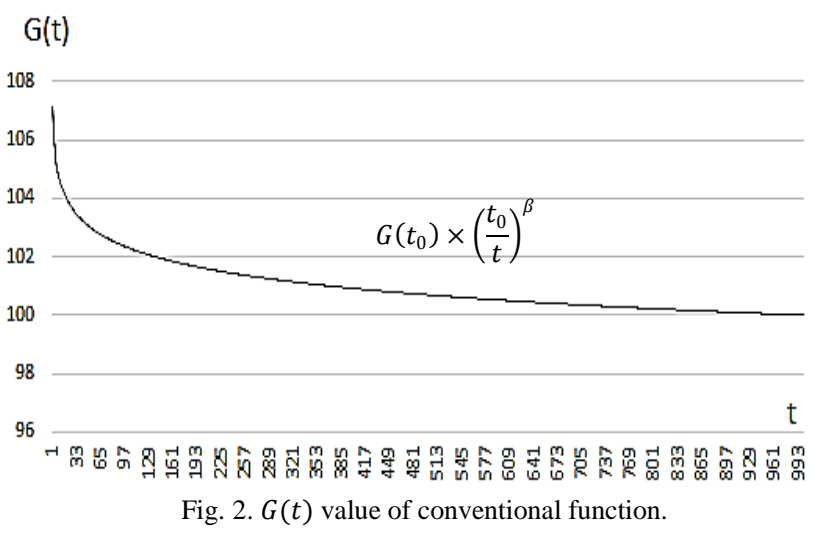

$\mathrm{G}(\mathrm{t})$

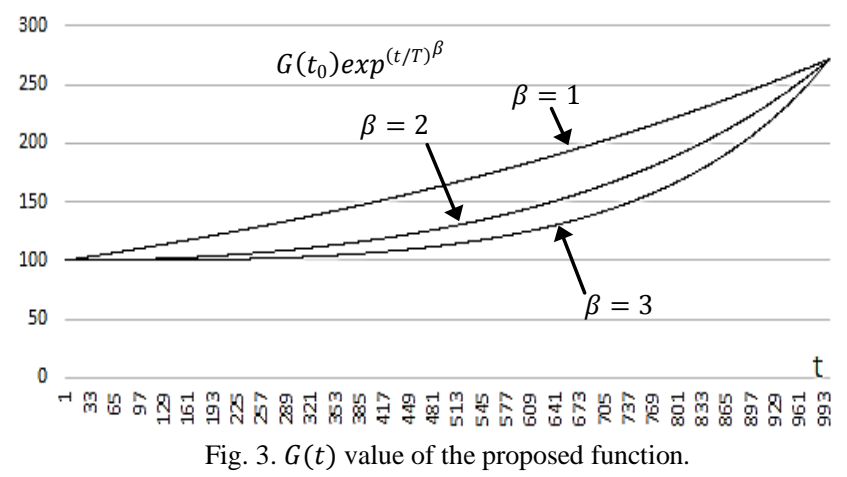

IV. COMPUTATIONAL EXPERIMENTS

In this section, we conduct the experiments in order to verify the performance of GSA based on the differences of gravitational coefficient functions. We perform the numerical experiments on four nonlinear benchmark functions, which consist of both unimodal and multimodal optimization problems. For the computational test purpose, all gravitational coefficient functions are written in VBA and executed on PC with Intel Core i5-7200U CPU @ 2.50 $\mathrm{GHz}$ processor and $8 \mathrm{~GB}$ RAM.

\section{A. Benchmark Functions}

Four benchmark functions in literature are selected. They can be divided into two categories; unimodal landscape functions and multimodal landscape functions. The unimodal landscape function in this study consists of Rosenbrock function while the multimodal landscape functions consist of Three-hump camel function, Griewank function, and Butterfly function. Theoretically, the multimodal landscape functions are not easy to find the global optimal solutions. Furthermore, it also depends on the starting point of the search. In this study, the starting points are generated randomly. Moreover, we test them on two dimensional function only, for the sake. Table I and Table II show the detailed formula of the functions, its search space boundary for this study, and their optimal solutions.

Table II shows the optimal solutions of the benchmark functions. Fig. 4-Fig. 7 illustrate two dimensional landscape to the benchmark functions of this study.

\begin{tabular}{ccc} 
& \multicolumn{2}{c}{ TABLE I: TEST FUNCTIONS } \\
\hline \hline $\begin{array}{c}\text { Function } \\
\text { name }\end{array}$ & Description & $\begin{array}{c}\text { Search } \\
\text { Range }\end{array}$ \\
\hline $\begin{array}{c}\text { Rosenbrock } \\
\text { Three-hump } \\
\text { camel }\end{array}$ & $f(X)=100\left(x_{2}-x_{1}^{2}\right)^{2}+\left(1-x_{1}\right)^{2}$ & {$[-10,10]^{2}$} \\
Griewank & $f(X)=2 x_{1}^{2}-1.05 x_{1}^{4}+\frac{x}{6}+x_{1} x_{2}+x_{2}^{2}$ & {$[-10,10]^{2}$} \\
Butterfly & $f(X)=\frac{\left(x_{1}^{2}+x_{2}^{2}\right)}{4000}-\cos x_{1} \cos \left(\frac{x_{2}}{\sqrt{2}}\right)+1$ & {$[-600,600]^{2}$} \\
\hline \hline
\end{tabular}

TABLE II: OPTIMAL SOLUTIONS OF THE TEST FUNCTIONS

\begin{tabular}{ccc}
\hline \hline Function & \multicolumn{2}{c}{ Optimal Solution } \\
\cline { 2 - 3 } name & $f\left(X^{*}\right)$ & $\left(x_{1}^{*}, x_{2}^{*}\right)$ \\
\hline Rosenbrock & 0 & $(1.1)$ \\
Three-hump camel & 0 & $(0,0)$ \\
Griewank & 0 & $(0,0)$ \\
Butterfly & -1 & $(-0.74775,341.5874)$ \\
\hline \hline
\end{tabular}

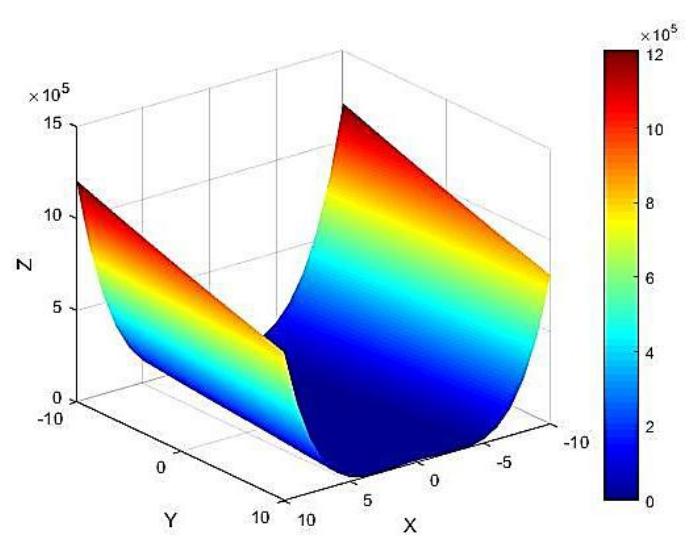

Fig. 4. Rosenbrock function.

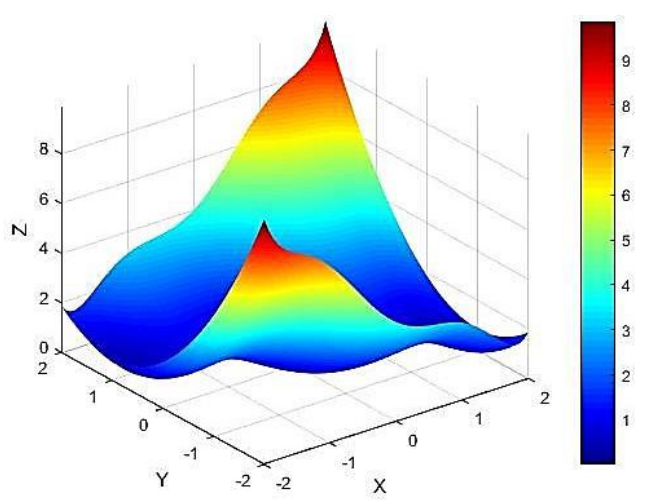

Fig. 5. Three-hump camel function.

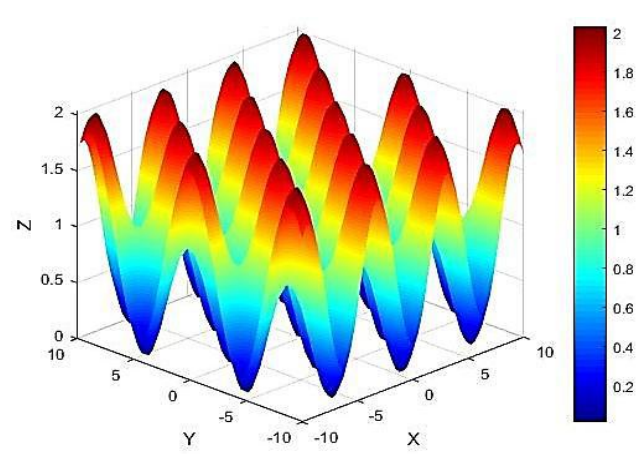

Fig. 6. Griewank function. 


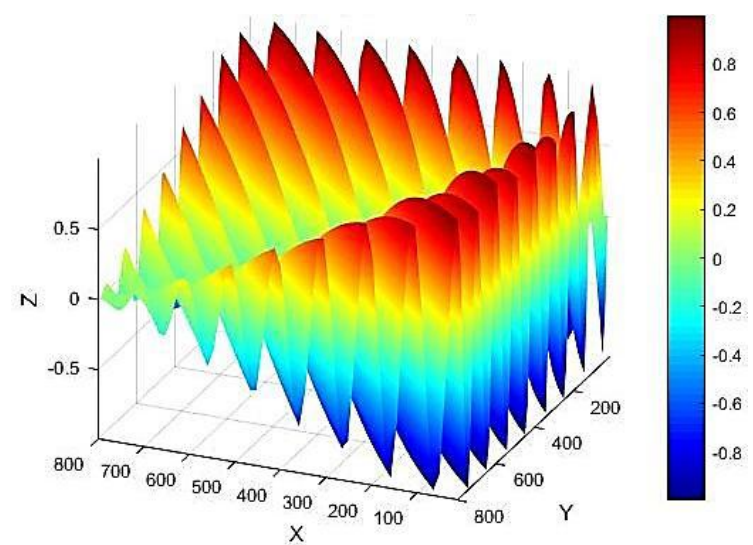

Fig. 7. Butterfly function.

\section{B. Parameter Settings}

The setting of parameters is significant for a computational experiment. We started with the literature review and conducted preliminary experiments.

For the conventional $G(t)$ function: $t_{0}$ equals $1000, \beta=$ 0.01 , and $G\left(t_{0}\right)=100$. For the proposed $G(t)$ function: $T$ equals $1000, \beta=2$, and $G\left(t_{0}\right)=100$. Additionally, the number of agents $\mathrm{N}$ is 50. As GSA falls into stochastics searching technique, we executed each combination of experiment for 30 independent runs in order to eliminate random discrepancy.

\section{RESUlTS AND DISCUSSION}

\section{A. Experimental Results}

All of the test results in terms of the mean optimal solutions and its standard deviation are summarized in Table III. The best results between two gravitational coefficient functions for each benchmark function are underlined. As shown in the table, the proposed $G(t)$ function outperforms the conventional one on all 4 benchmark functions. It yields the results closer to the optimal solution on both unimodal landscape function and multimodal landscape functions. Furthermore, it also yields the lower uncertainty of the results. This means that the proposed $G(t)$ is more reliable on the search course. However, statistically, we cannot claim that the proposed $G(t)$ is better than the conventional $G(t)$ because it concerns to the uncertainty of the results. Thus, we need to conduct a post-hoc analysis by using a statistical technique.

TABLE III: EXPERIMENTAL RESULTS

\begin{tabular}{|c|c|c|c|c|}
\hline \multirow{2}{*}{ Function name } & \multicolumn{2}{|c|}{ Conventional $G(t)$} & \multicolumn{2}{|c|}{ Proposed $G(t)$} \\
\cline { 2 - 5 } & Mean & Std. & Mean & Std. \\
\hline Rosenbrock & 1.190 & 1.590 & $\underline{0.510}$ & 0.676 \\
\hline Three-hump camel & 0.485 & 0.252 & $\underline{0.107}$ & 0.026 \\
\hline Griewank & 2.230 & 1.400 & $\underline{0.261}$ & 0.192 \\
\hline Butterfly & -0.9983 & 0.0014 & $\underline{-0.9998}$ & 0.0001 \\
\hline
\end{tabular}

\section{B. Statistical Test}

As the metaheuristics methods are based on a stochastic search procedure, their results are varied and founded by the chance; accordingly, in order to verify the performance systematically, we need to conduct a statistical method for attesting them. In this study, we deploy the two-sample t-
Test with $\alpha=0.05$ for each test function because there is only one factor, the gravitational coefficient function $G(t)$. The hypothesis testing is stated as below.

$H_{0}$ : The conventional $G(t)$ is not different from the proposed $G(t)$

$H_{1}$ : The conventional $G(t)$ is different from the proposed $G(t)$

The statement of the hypothesis testing above is called two-tail hypothesis test. By using a statistics package, we found that on all benchmark functions, the proposed $G(t)$ function is significantly difference from the conventional $G(t)$ function. The detailed test values and the decisions are drawn in Table IV.

TABLE IV: STATISTICAL TEST (TWO-TAIL TEST)

\begin{tabular}{|c|c|c|c|c|}
\hline \multirow{2}{*}{ File name } & \multicolumn{4}{|c|}{ T-Sample T-Test } \\
\cline { 2 - 5 } & T-Value & P-Value & DF & Decision \\
\hline Rosenbrock & 2.150 & 0.035 & 58 & Reject $H_{0}$ \\
\hline Three-hump camel & 7.130 & 0.000 & 58 & Reject $H_{0}$ \\
\hline Griewank & 7.630 & 0.000 & 58 & Reject $H_{0}$ \\
\hline Butterfly & 5.340 & 0.000 & 58 & Reject $H_{0}$ \\
\hline
\end{tabular}

Furthermore, in order to have a strong conclusion on the statistical testing, we deploy one-tail hypothesis test. The hypothesis statement is stated as below.

$H_{0}$ : The proposed $G(t)$ equals the conventional $G(t)$

$H_{1}$ : The proposed $G(t)$ is less than the conventional $G(t)$

Again, the statistics package is used. The results and decisions are drawn in Table V. We could claim that the proposed gravitational coefficient function enhances the performance of GSA over the conventional gravitational coefficient function.

TABLE V: STATISTICAL TEST (ONE-TAIL TEST)

\begin{tabular}{|c|c|c|c|c|}
\hline \multirow{2}{*}{ File name } & \multicolumn{4}{|c|}{ T-Sample T-Test } \\
\cline { 2 - 5 } & T-Value & P-Value & DF & Decision \\
\hline Rosenbrock & -2.150 & 0.018 & 58 & Reject $H_{0}$ \\
\hline Three-hump camel & -7.130 & 0.000 & 58 & Reject $H_{0}$ \\
\hline Griewank & -7.630 & 0.000 & 58 & Reject $H_{0}$ \\
\hline Butterfly & -5.340 & 0.000 & 58 & Reject $H_{0}$ \\
\hline
\end{tabular}

\section{Convergence Characteristics}

We are interested to observe the convergence behavior on both gravitational coefficient functions in order to understand their characteristics. Fig. 8 shows that on the Rosenbrock function, the conventional $G(t)$ converges to the optimal value faster than the proposed $G(t)$. However, the conventional one tends to be premature. It could not do the exploitation phase considerably; while, the proposed one could exploit the search satisfactorily.

The ability of exploitation in the second phase is manifest on the multimodal landscape functions. Fig. 9, Fig. 10, and Fig. 11 show the ability of the proposed $G(t)$ of this study. While the solutions founded by the conventional $G(t)$ stuck at some points of the search space, the proposed $G(t)$ could conduct the fine search and yield the better solution continuously. 


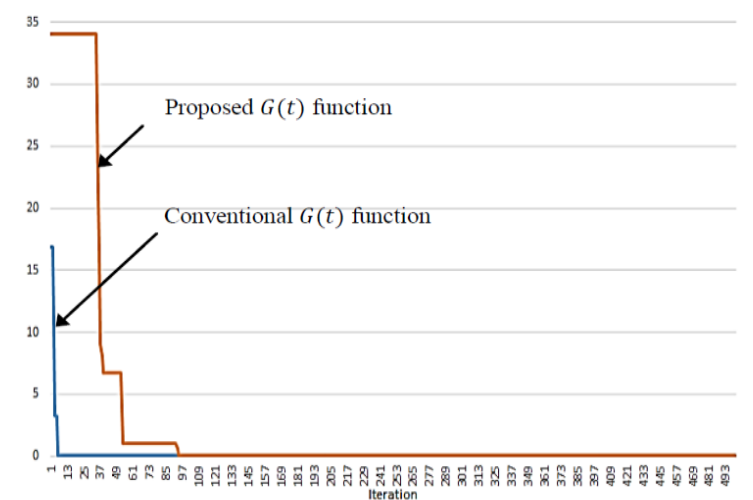

Fig. 8. Convergence characteristics on Rosenbrock function.

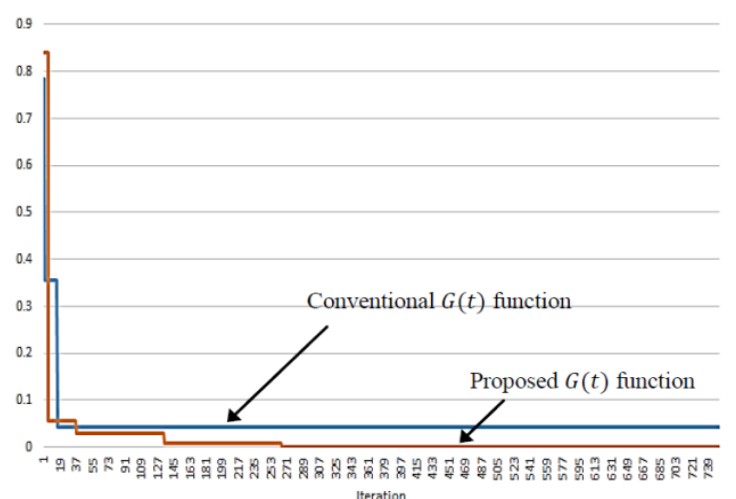

Fig. 9. Convergence characteristics on Three-hump camel function.

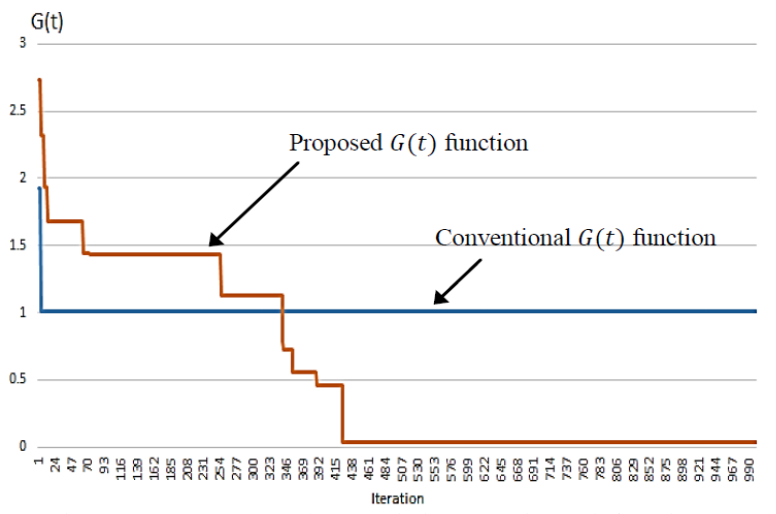

Fig. 10. Convergence characteristics on Griewank function.

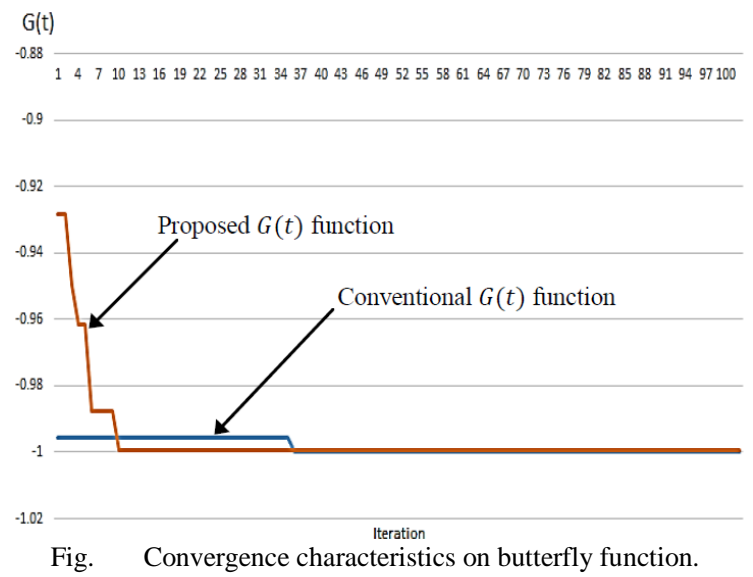

\section{CONCLUSION}

We proposed a novel gravitational coefficient function, $G(t)$. The function is essence on the step of the calculation of acceleration in Gravitational Search Algorithm (GSA). The proposed function is based on the idea of the exploration and the exploitation search phases. We proposed an exponential-like function which the value of $G(t)$ grow slowly in the first phase; then, it grows rapidly in the second phase. This enhances the search procedure conducts an exploration and then an exploitation systematically.

The proposed function was verified on four benchmark functions which consist of both unimodal landscape function and multimodal landscape functions. By comparing to the performance of the conventional $G(t)$, we found that the proposed $G(t)$ outperformed the conventional one. We also analyzed the results statistically. This could affirm that the novel function enhances the performance of GSA evidently. Moreover, we observed the convergence characteristics on both $G(t)$ functions. We found that the proposed $G(t)$ has slow convergence rate; however, the problem of premature convergence is avoidable. For the future research, we are interested to investigate the gravitational coefficient functions mathematically and prove it rigorously. More benchmark functions and more comparison tests are required.

\section{REFERENCES}

[1] Rashedi, "Gravitational search algorithm," M.Sc. thesis, Dept. Elect. Eng., Shahid Bahonar University of Kerman, Kerman, Iran, 2007.

[2] E. Rashedi, H. Nezamabadi-Pour, and S. Saryazdi, "GSA: A gravitational search algorithm," Information Science, vol. 179, no. 13, pp. 2232-2248, June 2009.

[3] M. B. Dowlatshahi and H. Nezamabadi-pour, "GGSA: A grouping gravitational search algorithm for data clustering," Engineering Applications of Artificial Intelligence, vol. 35, pp. 114-121, Nov. 2014.

[4] S. Ghavidel, J. Aghaei, K. M. Muttaqi, and A. Heidari, "Renewable energy management in a remote area using modified gravitational search algorithm," Energy, vol. 97, Feb. 2016.

[5] C. Saravanan and K. Srinivasan, "Optimal extraction of photovoltaic model parameters using gravitational search algorithm approach," Circuits and Systems, vol. 7, no. 11, pp. 3849-3861, Sep. 2016.

[6] M. Packiasudha, S. Suja, and J. Jerome, "A new cumulative gravitational search algorithm for optimal placement of FACT device to minimize system loss in the deregulated electrical power environment," International Journal of Electrical Power \& Energy Systems, vol. 84, pp. 34-46, Jan. 2017.

[7] A. Ahmad, S. F. Razali, Z. S. Mohamed, and A. El-shafie, "The application of artificial bee colony and gravitational search algorithm in reservoir optimization," Water Resources Management, vol. 30, no. 7, pp. 2497-2516, May 2016.

[8] P. K. Das, H. S. Behera, and B. K. Panigrahi, "A hybridization of an improved particle swarm optimization and gravitational search algorithm for multi-robot path planning," Swarm and Evolutionary Computation, vol. 28, pp. 14-28, Jun. 2016.

[9] A. A. R. Hosseinabadi, M. Kardgar, M. Shojafar, S. Shanshirband, and A. Abraham, "Gravitational search algorithm to solve open vehicle routing problem," Innovations in Bio-Inspired Computing and Applications, vol. 424, pp. 93-103, Springer, Cham, 2016.

[10] S. Yazdani, H. Nezamabadi-pour, and S. Kamyab, "A gravitational search algorithm for multimodal optimization," Swarm and Evolutionary Computation, vol. 14, pp. 1-14, Feb. 2014.

[11] T. Eldos and R. Al Qasim, "On the performance of the gravitational search algorithm," International Journal of Advanced Computer Science Applications, vol. 4, no. 3, pp. 74-78, Aug. 2013.

[12] B. Yin, Z. Guo, Z. Liang, and X. Yue, "Improved gravitational search algorithm with crossover," Computers \& Electrical Engineering, vol. 66, pp. 505-516, 2018.

[13] M. Khatibinia and S. Khosravi, "A hybrid approach based on an improved gravitational search algorithm and orthogonal crossover for optimal shape design of concrete gravity dams," Applied Soft Computing, vol. 16, pp. 223-233, 2014.

[14] J. Xiao, Y. Niu, P. Chen, S. C. Leung, and F. Xing, "An improved gravitational search algorithm for green partner selection in virtual enterprises," Neurocomputing, vol. 217, pp. 103-109, Dec. 2016.

[15] Y. Kumar and G. Sahoo, "A review on gravitational search algorithm and its applications to data clustering \& classification," 
International Journal of Intelligent Systems and Applications, vol. 6, no. 6, pp.79-93, May 2014.

[16] R. Mansouri, F. Nasseri, \& M. Khorrami "Effective time variation of $\mathrm{G}$ in a model universe with variable space dimension" Physics Letters A, vol. 259, pp. 194-200, Aug. 1999.

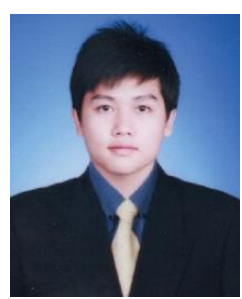

Pattrawet Tharawetcharak is a Ph.D. candidate in Engineering Management Program, Department of Industrial Engineering and Management, Faculty of Engineering and Industrial Technology, Silpakorn University, Nakhon Pathom, 73000 Thailand.

$\mathrm{He}$ is an instructor in General Education, Office of Learning Promotion and Provision Academic Services, Varaya Alongkorn Rajabhat University under The Royal Patronage, Pathumthani, Thailand. He has research interests such as manufacturing and logistics, clean technology, manufacturing supply chain and logistics systems design, optimization, production planning and control, process design, and computers in industrial engineering.

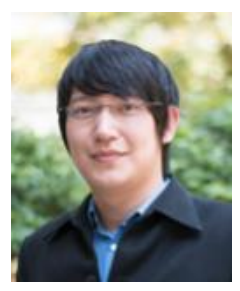

Thanathorn Karot is an instructor in the Department of Industrial Engineering and Management, Faculty of Engineering and Industrial Technology, Silpakorn University, Nakhon Pathom, Thailand. He has research interests such as sustainable engineering, manufacturing supply chain and logistics systems design, manufacturing systems, optimization, production planning and control, and computers in industrial engineering.

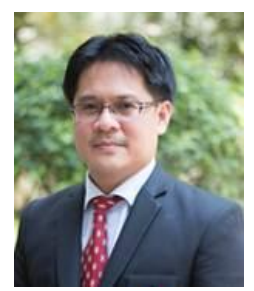

Choosak Pornsing is an instructor in the Department of Industrial Engineering and Management, Faculty of Engineering and Industrial Technology, Silpakorn University, Nakhon Pathom, Thailand. He has research interests such as sustainable engineering, manufacturing supply chain and logistics systems design, manufacturing systems, optimization, production planning and control, process design, and computers in industrial engineering.

Asst. Prof. Dr. Choosak Pornsing received an excellent oral presentation from International Conference on Control, Automation and Robotics in 2016 in Hong Kong and best practice in Industrial Engineering Technique Award from Engineering Institute of Thailand. 